Coopération politique et intégration régionale en Arctique (1996-2019) : construction d'une région, naissance, développement et remise en cause d'un nouvel espace politique régional

\title{
Camille Escudé-Joffres
}

\section{OpenEdition}

\section{Journals}

Édition électronique

URL : https://journals.openedition.org/cdg/7615

DOI : $10.4000 /$ cdg. 7615

ISSN : 2107-7266

\section{Éditeur}

UMR 245 - CESSMA

Référence électronique

Camille Escudé-Joffres, « Coopération politique et intégration régionale en Arctique (1996-2019)

construction d'une région, naissance, développement et remise en cause d'un nouvel espace politique régional », Carnets de géographes [En ligne], 15 | 2021, mis en ligne le 30 avril 2021, consulté le 11 juin 2021. URL : http://journals.openedition.org/cdg/7615 ; DOI : https://doi.org/10.4000/cdg.7615

Ce document a été généré automatiquement le 11 juin 2021.

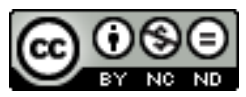

La revue Carnets de géographes est mise à disposition selon les termes de la Licence Creative Commons Attribution - Pas d'Utilisation Commerciale - Pas de Modification 4.0 International. 


\title{
Coopération politique et intégration régionale en Arctique (1996-2019) : construction d'une région, naissance, développement et remise en cause d'un nouvel espace politique régional
}

\author{
Camille Escudé-Joffres
}

1 Jusqu'à la fin de la Guerre froide, l'Arctique était avant toute chose un espace de potentiel affrontement entre l'URSS et le bloc occidental. En l'espace de trente ans, la construction de la gouvernance politique régionale s'est faite ex nihilo grâce à l'interaction de différentes échelles d'action politique correspondant à divers acteurs : États, organisations autochtones, gouvernements fédéraux et locaux, ONG, entreprises. L'Arctique est à présent impliquée dans divers mécanismes qui tendent vers une intégration régionale, ce processus d'intégration étant défini comme le processus par lequel la régularité et l'intensité des interactions entre les sociétés s'accroissent.

2 Notre travail de thèse est fondé sur une étude à la fois théorique et empirique. D'une part, notre thèse s'appuie sur la littérature constructiviste sur la région (Brenner 1997; Cox, 1998; Paasi, 1991; Mamadouh et Van der Wusten, 2008; Richard, 2018) et un travail d'analyse politique des structures de gouvernance arctique. D'autre part, nous avons mené une étude de cas empiriques avec de l'observation participante dans plusieurs conférences (Arctic Circle à Reykjavik, Arctic Frontiers à Tromsø) et cinquante entretiens menés auprès de représentants des États arctiques, d'États extérieurs à la région mais souhaitant intervenir dans la gouvernance régionale, de représentants politiques à d'autres échelles (provinciale, autochtone) ainsi que de membres des ONG et communautés épistémiques impliquées. 
Il s'agit dans notre thèse d'analyser la manière dont la gouvernance politique à toutes les échelles a construit, et continue à construire, l'espace arctique comme une région. La création de régions est conditionnée par les politiques, les cultures, les économies, les gouvernances, les représentations et les relations de pouvoir de manière contingente (Paasi, 2010) et l'existence des régions est précédée par celle de "regionbuilders", c'est-à-dire d'acteurs politiques qui créent des régions comme projet politique. En Arctique, c'est avant tout le régionalisme institutionnel qui dessine les limites d'une région politique depuis la création du Conseil de l'Arctique en 1996, mais il existe une "mosaïque de coopération» (Young, 2005) impliquant de nombreuses autres instances. En Arctique comme ailleurs, parce que ce sont des volontés politiques à géométrie variable qui ont permis l'intégration régionale, la région a des limites floues, mouvantes et contestées.

4 À ce titre, les multiples mécanismes de coopération qui dessinent un réseau de gouvernance de l'espace arctique participent à la construction et à l'organisation de cette région. Walter Mattli avait déjà montré que, dans le cas de l'Europe, la coopération politique peut mener à l'intégration régionale (Mattli, 1999). On retrouve ce même type de processus en Arctique: la protection de l'environnement joue notamment un rôle de levier dans le processus de régionalisation. En effet, le processus d'intégration régionale implique l'établissement d'institutions comme le Conseil de l'Arctique qui ont pour mission d'atteindre les objectifs de l'intégration et de les maintenir dans la durée, et constitue à ce titre le « noyau dur » de l'intégration (Girault, 2018), ne serait-ce qu'en offrant aux acteurs un cadre de relations formelles ou informelles. La gouvernance régionale contribue dans ce cas à améliorer l'intégration régionale, qui augmente au fur et à mesure que les acteurs qui bénéficient de la coopération œuvrent à la renforcer. La géographie commune de l'Arctique ainsi que les interactions régulières et intenses entre acteurs, à de divers niveaux, ont généré une forme de cohésion politique régionale.

5 La gouvernance régionale arctique dessine ainsi des limites d'une région politique qui sont celles du cercle polaire, c'est-à-dire des territoires arctiques situés au sein des huit Etats membres du Conseil de l'Arctique (Canada, Groenland avec le Danemark, EtatsUnis, Finlande, Islande, Suède, Norvège, Russie). Or dans un contexte d'intérêt mondial pour la région arctique, des acteurs issus du monde entier (pays de l'Union européenne ou pays asiatiques comme la Chine, l'Inde ou le Japon devenus membres observateurs du Conseil de l'Arctique en 2013) s'immiscent dans les instances politiques, dessinant une région institutionnelle aux limites à géométrie variable. D'une part, le cercle des acteurs revendiquant une place dans les décisions de la région arctique s'agrandit. D'autre part, ces acteurs extérieurs à la région se heurtent à la volonté des acteurs arctiques de les maintenir hors des décisions régionales, et ce même si plusieurs de ces acteurs ne s'impliquent guère dans les activités des groupes de travail du Conseil de l'Arctique (Babin et Lasserre, 2019) ou nourrissent des attentes excessives (Rottem, 2020). Nombreux sont les membres observateurs du Conseil de l'Arctique qui jugent que le modèle actuel de gouvernance restreint atteint ses limites et le remettent en cause. Ces mêmes observateurs déploient des trésors d'imagination pour contourner les institutions politiques ou inventer de nouvelles formes d'action politique pour faire entendre leur voix sous la forme de forums alternatifs. Sous les coups de boutoir des États extérieurs à l'Arctique, des gouvernements fédéraux comme l'Ecosse et des ONG, 
les limites de la participation à la gouvernance de la région arctique deviennent mouvantes, se déplaçant toujours plus vers des espaces extra- ou infra-polaires.

6 Les conférences internationales arctiques sont ainsi actuellement des plateformes privilégiées de l'expression d'acteurs ordinairement mis de côté de la coopération formelle en Arctique. L'exemple de la conférence internationale " Arctic Circle » qui se tient tous les mois d'octobre depuis 2013 à Reykjavik est représentatif à cet égard. Que ce soit pour les entreprises, les États non riverains, voire non-Arctiques ou encore les gouvernements infranationaux, ils permettent une coopération plus ouverte. Ces rencontrent se font à travers des réunions, des sessions en petit comité, ou des rencontres plus informelles. "Arctic Circle», "plateforme démocratique et ouverte » auto-proclamée s'oppose de manière tacite aux organisations fermées comme le Conseil de l'Arctique en se voulant «le plus grand rassemblement international sur l'Arctique ». De multiples villes, pays ou gouvernements fédéraux ont également accueilli un forum délocalisé : la Chine, la Corée du Sud, Singapour, les États-Unis. Du côté des territoires non-étatiques, les îles Féroé, l'Écosse, le Québec, le Groenland ont également accueilli ce type d'évènement. La conférence "Arctic Circle» est même considérée pour certains comme un format politique alternatif pour les exclus du Conseil de l'Arctique. Cela illustre l'élargissement du cadre de la régionalisation arctique, géographiquement avec cette extension auréolaire vers l'Asie et politiquement, en incluant des acteurs non étatiques

7 Ce processus lent et inégal d'institutionnalisation de la région arctique ne marque pas l'avènement d'un niveau régional de gouvernement. Les institutions contribuent cependant à densifier la connexion fonctionnelle ou du moins discursive entre les habitants de la région. Alors que l'efficacité d'une institution est généralement comprise en fonction de sa capacité à résoudre le problème spécifique pour lequel elle était mise en place - pour le Conseil de l'Arctique, protéger l'environnement - une autre manière d'évaluer les institutions arctiques serait d'analyser jusqu'à quel point elles contribuent à la construction de l'Arctique comme région politique. Cela n'implique pas forcément que les territoires de cette région doivent être autosuffisants en termes économiques, politiques ou autre. Cela signifie que les individus, groupes et représentants d'États impliqués lisent les problèmes et opportunités dans la région à travers un prisme régional, et que les personnes extérieures reconnaissent également la région comme un ensemble. À ce titre, l'Arctique comme projet politique semble sur la bonne voie. 
Figure 1. Une extension politique de l'Arctique

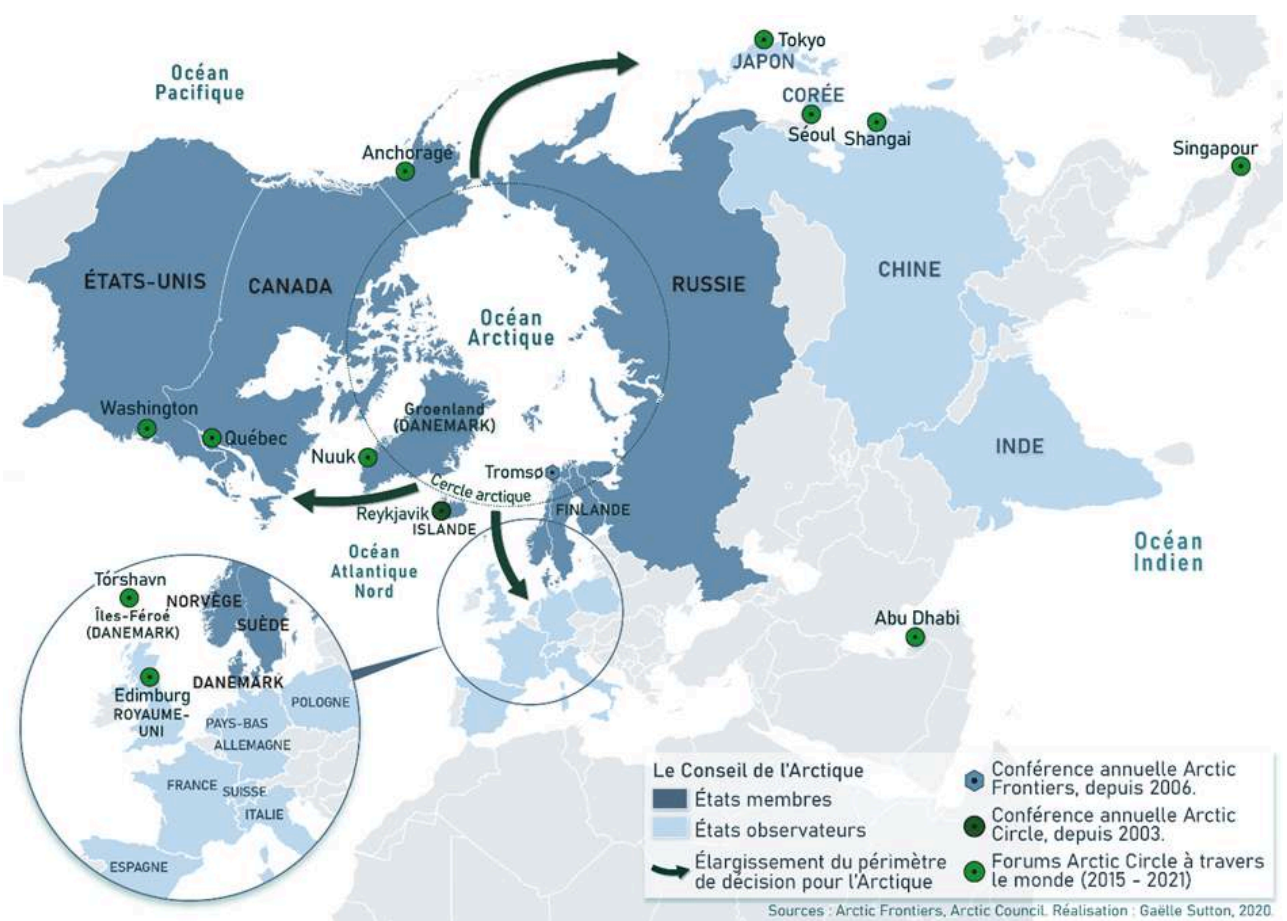

Source : Escudé-Joffres, 2020.

Lien électronique de la thèse

https://hal.inria.fr/tel-02980869

\section{Discipline}

Science politique ; Géographie

\section{Directeur}

Guillaume Devin, CERI

\section{Université}

Sciences Po, CERI

Membres du jury de thèse, soutenue le 18 septembre 2020

Mme Cécile Crespy, Professeure des universités, Institut d'études politiques de Toulouse (rapporteure)

M. Guillaume Devin, Professeur des universités, Institut d'études politiques de Paris

M. Frédéric Lasserre, Professeur titulaire, Université Laval

Mme Virginie Mamadouh, Professeure, Universiteit van Amsterdam

M. Franck Petiteville, Professeur des universités, Institut d'études politiques de Grenoble

M. Yann Richard, Professeur des universités, Université Paris 1 Panthéon Sorbonne (rapporteur)

Situation professionnelle à l'issue de la thèse

ATER à Sciences Po 


\section{BIBLIOGRAPHIE}

Babin J., Lasserre F. (2019), « Asian states at the Arctic Council : perceptions in Western States », Polar Geography, vol. 42, no. 3, pp. 145-159. https://doi.org/10.1080/1088937X.2019.1578290.

Brenner N. (1997), « State Territorial Restructuring and the Production of Spatial Scale », Political Geography, vol. 16, no. 4, pp. 273-306. https://doi.org/10.1016/S0962-6298(96)00003-0.

Cox K. R. (1998), « Spaces of dependence, spaces of engagement and the politics of scale, or : looking for local politics », Political Geography, vol. 17, no. 1, pp. 1-23. https://doi.org/10.1016/ S0962-6298(97)00048-6.

Girault C. (2018), « Institutions régionales : deux modèles principaux pour un ensemble multiforme ", in Dictionnaire de la régionalisation du monde, Neuilly-sur-Seine, Atlande, pp. 60-62.

Mamadouh V., Van der Wusten H. (2008), « The European level in EU governance : territory, authority and trans-scalar networks », GeoJournal, vol. 72, no. 1, pp. 19-31. https://doi.org/ 10.1007/s10708-008-9162-8.

Paasi A. (1991), « Deconstructing Regions : Notes on the Scales of Spatial Life », Environment and Planning A, vol. 23, no. 2, pp. 239-256. https://doi.org/10.1068/a230239.

Paasi A. (2010), « Regions Are Social Constructs, but Who or What 'constructs" Them ? Agency in Question ", Environment and Planning A, vol. 42, no. 10, pp. 2296-2301. https://doi.org/10.1068/ a42232.

Richard Y. (2018) « Nouveau régionalisme : un renouvellement de la recherche sur l'intégration régionale ", in Dictionnaire de la régionalisation du monde, Neuilly-sur-Seine, Atlande, pp. 184-186.

Rottem S. V. (2020), The Arctic Council : Between Environmental Protection and Geopolitics, Palgrave Pivot. https://doi.org/10.1007/978-981-13-9290-0.

Young O. R. (2005), « Governing the Arctic : From Cold War Theater to Mosaic of Cooperation ». Global Governance; vol. 11, no. 1, pp. 9-15.

\section{INDEX}

Thèmes : Carnets de soutenances 
AUTEUR

CAMILLE ESCUDÉ-JOFFRES

Docteure en géographie et en sciences politiques, ATER à Sciences Po.

camille.escude[at]sciencespo.fr. 